\title{
On the stability of planar step shock fronts in multi-dimensional spaces
}

\author{
By Taira ShIRota
}

(Received March 26, 1982)

\section{Introduction.}

In this note we study the equations of 2-dimensional isentropic compressible flow

$$
\frac{\partial}{\partial t}\left(\begin{array}{c}
\rho w_{1} \\
\rho w_{2} \\
\rho
\end{array}\right)+\frac{\partial}{\partial x_{1}}\left(\begin{array}{c}
\rho w_{1}^{2}+P \\
\rho w_{1} w_{2} \\
\rho w_{1}
\end{array}\right)+\frac{\partial}{\partial x_{2}}\left(\begin{array}{c}
\rho w_{1} w_{2} \\
\rho w_{2}^{2}+P \\
\rho w_{2}
\end{array}\right)=0,
$$

where ${ }^{t}\left(w_{1}, w_{2}\right)$ is the velocity, $\rho$ is the density and $P=P(\rho)$ is a well defined function of $\rho(>0)$, with $P_{\rho}(\rho)>0$.

Since Lax had developed mathematical theory of system of hyperbolic conservation laws ([6]), 1-dimensional shock fronts were investigated by many mathematicians, but multi-dimensional cases were treated by a few authors [[2], [3], [9] also [13], [14]). Recently Majda has investigated the stability of multi-dimensional shock fronts and obtained the local in time shock front solutions under the uniform stability condition with respect to the linearized problem. ([7], [8]).

The problem considered in this note is what phenomena may occur if the condition mentioned above is replaced by the weak stability. Because of difficulty to obtain exact answer with mathematical rigor, here we shall treat our problem as a perturbation one in the simplest form.

Let us the nonconservative variables $U={ }^{t}\left(w_{1}, w_{2}, P\right)$. Then equations (1. 1) become

$$
\left(\begin{array}{ll}
\rho & \\
& \rho \\
& \frac{1}{\rho c^{2}}
\end{array}\right) \frac{\partial U}{\partial t}+\left(\begin{array}{ccc}
\rho w_{1}, & 0, & 1 \\
0, & \rho w_{1}, & 0 \\
1, & 0, & \frac{w w_{1}}{\rho c^{2}}
\end{array}\right) \frac{\partial U}{\partial x_{1}}+\left(\begin{array}{ccc}
\rho w_{2}, & 0, & 0 \\
0, & \rho w_{2}, & 1 \\
0, & 1, & \frac{w w_{2}}{\rho c^{2}}
\end{array}\right) \frac{\partial U}{\partial x_{2}}=0,
$$

where $c^{2}=P_{\rho}(\rho)$.

Let $U^{0 \pm}$ be constant step states :

$$
{ }^{t}\left(w_{1}^{0+}, w_{2}^{0+}, P^{0+}\right) \quad \text { for } \quad x_{1} \geqq \sigma t,
$$




$$
{ }^{t}\left(w_{1}^{0-}, w_{2}^{0-}, P^{0-}\right) \quad \text { for } \quad x_{1} \leqq \sigma t,
$$

where the Rankine-Hugoniot conditions,

$$
\begin{aligned}
& w_{2}^{-}=w w_{2}^{+}=w_{2}, \\
& -\sigma[\rho]+\left[\rho w_{1}\right]=0, \\
& -\sigma\left[\rho w_{1}\right]+\left[\rho w_{1}^{2}+P(\rho)\right]=0
\end{aligned}
$$

are assumed to be satisfied with the basic shock speed $\sigma$. We also assume that Lax's 1-shock inequalities,

$$
\begin{aligned}
w_{1}^{+}-c^{+}<\boldsymbol{\sigma} & <w_{1}^{-}-c^{-}, \\
\boldsymbol{\sigma} & <w_{1}^{+}
\end{aligned}
$$

are satisfied for $U^{0 \pm}$.

We let $U^{ \pm}=U^{0 \pm}+U^{ \pm \pm}$and $\beta=\sigma t+\phi$ denote the perturbed shock front solution of $\left(1,1^{\prime}\right)$ such that for some $T, U^{ \pm} \in H^{s}\left(G^{ \pm}\right), \phi \in H^{s+1}\left((0, T) \times R^{1}\right)$,

$$
\left\|U^{\prime \pm}\right\|_{s, G^{ \pm}} \leqq k_{0}, \quad\left\langle\langle\phi\rangle_{s+1,(0, T) \times R^{1}} \leqq k_{0},\right.
$$

$\operatorname{Supp}_{x}\left(U^{\prime \pm}\right)$ and $\operatorname{Supp}_{x_{2}}(\phi)$ are contained in $\{|x| \leqq 1\}$ and its intersection with $\left\{x_{1}=0\right\}$ respectively. Here $k_{0}$ is a sufficiently small constant depending only on $\left\{U^{ \pm \pm}, \sigma\right\}, s=2\left[\frac{n}{2}\right]+7$ (here $n=2$ ),

$$
G^{ \pm}=\left\{(t, x) \mid \beta\left(t, x_{2}\right) \gtrless x_{1} \quad \text { for } \quad t \in(0, T)\right\},
$$

$\|U\|_{s, \Omega}$ is the Sobolev norm of order $s$ of $U$ relative to $\Omega$ and $\left\langle\langle U\rangle_{s,(0, T) \times R^{1}}\right.$ is the 2-dimensional sobolev norm.

For initial data $\frac{\partial^{i} U^{\prime \pm}}{\partial t^{i}}(0, x), \frac{\partial^{i+1} \phi}{\partial t^{i+1}}\left(0, x_{2}\right)(0 \leqq i \leqq s+l)$ we impose the following conditions :

$l$ is some positive integer, $\phi\left(0, x_{2}\right)=0$, the supports of these functions $\subset \frac{1}{2}$-disks with 0 as the center, $H^{s+l-i}$-norms in the definition domains of these functions are not greater than some constant $k_{0}^{\prime}$ for $i \leqq s+l$, all of functions with $i \leqq s-1$ vanish on $\left\{x_{1}=0\right\}, \frac{\partial^{i} U^{\prime \pm}}{\partial t^{i}}(0, x)$ with $i \leqq s-1$ satisfy the compatibility conditions up to order $s-1$ on $\left\{x_{1} \gtrless 0\right\}$, respectively.

Hereafter these functions also are denoted by $\left\{U^{\prime \pm}, \phi\right\}$.

To state our theorem, we introduce the following

DEFINITION. We say the basic states $\left\{U^{0 \pm}, \sigma t\right\}$ to be stable in $L^{2}$-sense if (i) for any non-vanishing initial data mentioned above with sufficiently 
large $l$ and small $k_{0}^{\prime}$ there is a unique shock front solution satisfying the conditions (1.4) for some $T$, such that

$$
\int\left|U^{\prime}\right|^{2}(t, x) d x \leqq c \int\left|U^{\prime}\right|^{2}(0, x) d x, \quad \text { for } t \in[0, T] .
$$

Here $\int\left|U^{\prime}\right|^{2}(0, x) d x=\iint_{x_{1}>0}\left|U^{\prime+}\right|^{2}(0, x) d x_{1} d x_{2}+\iint_{x_{1}<0}\left|U^{\prime-}\right|^{2}(0, x) \cdot d x_{1} d x_{2}$.

Furthermore $c$ depends only on $k_{0}^{\prime}, l, T$.

ThEOREM. The basic states $\left\{U^{0 \pm}, \sigma t\right\}$ are stable in $L^{2}$-sense if and only if the linearized problem with respect to $\left\{U^{0+}, \sigma\right\}$ is uniformly stable.

The proof of Theorem is based on our investigation of the linear hyperbolic mixed problem. In fact we shall reduce our problem to examine the vanishing order of reflection coefficients of the linearized problem. ([1], [10]). Therefore in order to obtain an analogous conclusion of Theorem, we may weaken conditions in Definition of the stability in $L^{2}$-sense. But in (ii) only relevant physical quantities are appeared in $L^{2}$-sense and it seems to me that the definition is also significant for the nonlinear problems.

After preliminaries, in section 3 and 4 we give the proof of Theorem. Finally, in section 5 we discuss about its significance and an example.

\section{Preliminaries.}

We let $(1.1)$ and $\left(1.1^{\prime}\right)$ rewrite as follows:

$$
\begin{aligned}
& \frac{\partial}{\partial t} F_{0}(U)+\frac{\partial}{\partial x_{1}} F_{1}(U)+\frac{\partial}{\partial x_{2}} F_{2}(U)=0, \\
& A_{0}(U) \frac{\partial U}{\partial t}+A_{1}(U) \frac{\partial U}{\partial x_{1}}+A_{2}(U) \frac{\partial U}{\partial x_{2}}=0
\end{aligned}
$$

respectively. Then our problem is to find a set of functions $\left\{U^{+}, U^{-}, \beta\right\}$ called a shock front solution such that

$$
\begin{aligned}
& A_{0}\left(U^{ \pm}\right) \frac{\partial U^{ \pm}}{\partial t}+A_{1}\left(U^{ \pm}\right) \frac{\partial U^{ \pm}}{\partial x_{1}}+A_{2}\left(U^{ \pm}\right) \frac{\partial U^{ \pm}}{\partial x_{2}}=0 \\
& \text { in }\left\{x_{1} \gtrless \beta\left(t, x_{2}\right)\right\} \quad \text { respectively, } \\
& n_{t}\left(F_{0}\left(U^{+}\right)-F_{0}\left(U^{-}\right)\right)+n_{1}\left(F_{1}\left(U^{+}\right)-F_{1}\left(U^{-}\right)\right)+n_{2}\left(F_{2}\left(U^{+}\right)-F_{2}\left(U^{-}\right)\right)=0 \\
& \text { on }\left\{x_{1}=\beta\left(t, x_{2}\right)\right\},
\end{aligned}
$$

where $\left(n_{t}, n_{1}, n_{2}\right)$ is the time space normal of the surface $\left\{x_{1}=\beta\left(t, x_{2}\right)\right\}$. Let us denote by $U^{ \pm}=U^{0 \pm}+U^{\prime \pm}, \beta=\sigma t+\phi$ a perturbed solution for $t \geqq 0$. Then via the coordinate transformation: 


$$
\begin{aligned}
& \tilde{x}_{1}=x_{1}-\beta\left(t, x_{2}\right), \\
& \tilde{x}_{2}=x_{2}, \\
& \tilde{t}=t,
\end{aligned}
$$

the equations satisfied by $\left\{U^{\prime \pm}, \phi\right\}$ may be written as follows,

$(2.1)^{ \pm}$

$$
\begin{aligned}
A_{0}\left(U^{0 \pm}\right. & \left.+U^{\prime \pm}\right) \frac{\partial U^{ \pm}}{\partial t}+\left(A_{1}\left(U^{0 \pm}+U^{\prime \pm}\right)-\sigma A_{0}\left(U^{0 \pm}+U^{\prime \pm}\right)\right) \frac{\partial U^{\prime \pm}}{\partial x_{1}} \\
& +A_{2}\left(U^{0 \pm}+U^{\prime \pm}\right) \frac{\partial U^{\prime \pm}}{\partial x_{2}}-\left(\phi_{t} A_{0}\left(U^{0}+U^{\prime \pm}\right)\right. \\
& \left.+\phi_{x_{2}} A_{2}\left(U^{0}+U^{\prime \pm}\right)\right) \frac{\partial U^{\prime \pm}}{\partial x_{1}}=0
\end{aligned}
$$

in $\left\{x_{1}>0\right\}$ and $\left\{x_{1}<0\right\}$ respectively,

$$
\begin{gathered}
\sigma\left(F_{0}\left(U^{0+}+U^{\prime+}\right)-F_{0}\left(U^{0-}+U^{\prime-}\right)\right)-\left(F_{1}\left(U^{0+}+U^{\prime+}\right)-F_{1}\left(U^{0-}+U^{-}\right)\right) \\
+\phi_{t}\left(F_{0}\left(U^{0+}+U^{\prime+}\right)-F_{0}\left(U^{0-}+U^{\prime-}\right)\right) \\
+\phi_{x_{2}}\left(F_{2}\left(U^{0+}+U^{\prime+}\right)-F_{2}\left(U^{0-}+U^{\prime-}\right)\right) \\
=0 \quad \text { on }\left\{x_{1}=0\right\} .
\end{gathered}
$$

Since the basic states $U^{0^{ \pm}}$are constant, the linearized equations with respect to $\left\{U^{0 \pm}, \sigma\right\}$ are of the form :

$$
\begin{gathered}
A_{0}\left(U^{0^{ \pm}}\right) \frac{\partial U^{\prime \pm}}{\partial t}+\left(A_{1}\left(U^{0^{ \pm}}\right)-\sigma A_{0}\left(U^{0 \pm}\right)\right) \frac{\partial U^{\prime \pm}}{\partial x_{1}}+A_{2}\left(U^{0 \pm}\right) \frac{\partial U^{\prime \pm}}{\partial x_{2}}=0 \\
\text { in }\left\{x_{1}>0\right\} \text { and }\left\{x_{1}<0\right\} \quad \text { respectively, } \\
\phi_{t}\left(F_{0}\left(U^{0+}\right)-F_{0}\left(U^{0-}\right)\right)+\phi_{x_{2}}\left(F_{2}\left(U^{0+}\right)-F_{2}\left(U^{0-}\right)\right) \\
+\left(\sigma F_{0}^{\prime}\left(U^{0+}\right)-F_{1}^{\prime}\left(U^{0+}\right)\right) U^{\prime+}-\left(\sigma F_{0}^{\prime}\left(U^{0-}\right)-F_{1}^{\prime}\left(U^{0-}\right)\right) U^{\prime-}=0 \\
\text { on }\left\{x_{1}=0\right\} .
\end{gathered}
$$

Here $F_{0}^{\prime}\left(U^{0}\right) U^{\prime}$ is the Fréchet derivative at $U^{0}$ of $F_{0}\left(U^{0}+U^{\prime}\right)$ denoted usually by $F_{0}^{\prime}\left(U^{0} ; U^{\prime}\right)$ and $\left\{U^{\prime \pm}, \phi\right\}$ are unknown functions.

Following Majda ([7]) we now describe the Lopaninskii determinant of $\left(2.1^{\prime}\right)$ and $\left(2.2^{\prime}\right)$. First we note that $U^{\prime}$ is determined by the equation $\left(2.1^{\prime}\right)^{-}$ and the initial Cauchy data, for (1.3) is valid. Thus we may take $\left\{U^{\prime+}, \phi\right\}$ as unknown functions. The eigenvalues and corresponding eigenvectors of $A_{0}\left(U^{0+}\right)^{-1}\left(A_{1}\left(U^{0+}\right)-\sigma A_{0}\left(U^{0+}\right)\right)$ are given as follows : we set $w_{i}^{0}=w_{i}^{0+}, \rho^{0}=\rho^{0+}$ and $c^{0}=P_{\rho}\left(\rho^{0}\right)^{\frac{1}{2}}$, then 


$$
\begin{aligned}
& \lambda_{1}^{0}=w_{1}^{0}-\sigma-c^{0}<0, \quad \lambda_{2}^{0}=w_{1}^{0}-\sigma>0, \\
& \lambda_{3}^{0}=w_{1}^{0}-\sigma+c^{0}>\lambda_{2}^{0}, \\
& \gamma_{1}=\frac{1}{\sqrt{2}} t\left(-1,0, c^{0} \rho^{0}\right), \gamma_{2}={ }^{t}(0,1,0) \text { and } \gamma_{3}=\frac{1}{\sqrt{2}} t\left(1,0, c^{0} \rho^{0}\right) .
\end{aligned}
$$

We let $N_{0}$ be the matrix $\left(\gamma_{2}, \gamma_{3}, \gamma_{1}\right)$. Then direct calculations yield:

$$
\begin{aligned}
& N_{0}^{-1}=\left(\begin{array}{ccc}
0, & 1, & 0 \\
\frac{1}{\sqrt{2}}, & 0, & \frac{1}{\sqrt{2} c^{0} \rho^{0}} \\
-\frac{1}{\sqrt{2}}, & 0, & \frac{1}{\sqrt{2} c^{0} \rho^{0}}
\end{array}\right), \\
& N_{0}^{-1}\left(\begin{array}{c}
w_{1}^{\prime} \\
w_{2}^{\prime} \\
P^{\prime}
\end{array}\right)=\left(\begin{array}{c}
w_{2}^{\prime} \\
\frac{1}{\sqrt{2}}\left(\frac{P^{\prime}}{c^{0} \rho^{0}}+w_{1}^{\prime}\right. \\
\frac{1}{\sqrt{2}}\left(\frac{P}{c^{0} \rho^{0}}-w_{1}^{\prime}\right.
\end{array}\right) \equiv\left(\begin{array}{c}
v_{1} \\
v_{2} \\
v_{3}
\end{array}\right),
\end{aligned}
$$

where $\left(w_{1}^{\prime}, w_{2}^{\prime}, P^{\prime}\right)=\left(w_{1}^{\prime+}, w_{2}^{\prime+}, P^{\prime+}\right)$.

The equations for $V={ }^{t}\left(v_{1}, v_{2}, v_{3}\right)$ become as follows :

$$
\begin{gathered}
D_{x_{1}} V-M\left(D_{t}, D_{x_{2}}\right) V=0, \\
M\left(D_{t}, D_{x_{2}}\right)=\left(\begin{array}{ccc}
\frac{-1}{w_{1}^{0}-\sigma} \tilde{D}_{t} & , \frac{-c^{0}}{\sqrt{2}\left(w_{1}^{0}-\sigma\right)} D_{x_{2}}, \frac{-c^{0}}{\sqrt{2}\left(w_{1}^{0}-\sigma\right)} D_{x_{2}} \\
\frac{-c^{0}}{\sqrt{2}\left(w_{1}^{0}-\sigma+c^{0}\right)} D_{x_{2}}, \frac{-1}{w_{1}^{0}-\sigma+c^{0}} \tilde{D}_{t} & 0 \\
\frac{-c^{0}}{\sqrt{2}\left(w_{1}^{0}-\sigma-c^{0}\right)} D_{x_{2}}, & 0 & \frac{-1}{w_{1}^{0}-\sigma-c^{0}} \tilde{D}_{t}
\end{array}\right),
\end{gathered}
$$

where $D_{x_{1}}=\frac{1}{i} \frac{\partial}{\partial x_{1}}$ and $\tilde{D}_{t}=D_{t}+w_{2}^{0} D_{x_{2}}$.

The boundary conditions for $V$ on $\left\{x_{1}=0, t \geqq 0\right\}$ are :

$$
\begin{aligned}
& B=\left(\begin{array}{c}
\tilde{D_{t}}, 0,-\alpha D_{x_{2}} \\
0,1,-\beta
\end{array}\right), B V=0 \text { on } x_{1}=0, \\
& \alpha=\frac{\left(P\left(\rho^{0+}\right)-P\left(\rho^{0-}\right)\right)\left(w_{1}^{0}-\sigma-c^{0}\right)}{\left(\rho^{0+}-\rho^{0-}\right)\left(w_{1}^{0}-\sigma\right) \sqrt{2}\left(w_{1}^{0}-\sigma+c^{0}\right)}<0, \\
& \beta=-\left(\frac{c^{0}-\left(w_{1}^{0}-\sigma\right)}{c^{0}+\left(w_{1}^{0}-\sigma\right)}\right)^{2} \quad \text { and } 0>\beta>-1 .
\end{aligned}
$$


The boundary conditions for $\phi$ on $\left\{x_{1}=0, t \geqq 0\right\}$ are :

$$
\begin{aligned}
D_{x_{2}} \phi & =(-i) \frac{\rho^{0}\left(w_{1}^{0}-\sigma\right)}{P\left(\rho^{0+}\right)-P\left(\rho^{0-}\right)} v_{1}, \\
D_{t} \phi & =i\left\{\left(\frac{\left(w_{1}^{0}-\sigma\right) c^{0} \rho^{0}}{\sqrt{2}\left(\rho^{0+}-\rho^{0-}\right) c^{0}}+\frac{\rho^{0}}{\sqrt{2}\left(\rho^{0+}-\rho^{0-}\right)}\right) v_{2}\right. \\
& \left.+\left(\frac{\left(w_{1}-\sigma\right) c^{0} \rho^{0}}{\sqrt{2}\left(\rho^{0+}-\rho^{0-}\right) c^{0}}-\frac{\rho^{0}}{\sqrt{2}\left(\rho^{0+}-\rho^{0-}\right)}\right) v_{3}\right\} .
\end{aligned}
$$

Since $\phi\left(0, x_{2}\right)=0, \phi$ is determined from $V$.

Let the Fourier transformation of $f\left(t, x_{2}\right)$ be the following form :

$$
\hat{f}(\tau, \omega)=\int e^{-i\left(\tau t+\omega x_{2}\right)} f\left(t, x_{2}\right) d t d x_{2} .
$$

Then eigenvalues and the corresponding eigenvectors of $M(\tilde{\tau}, \omega)$ are: for $\tau=\eta-i \gamma$ and $\tilde{\tau}=\tau+\omega_{2}^{0} \omega\left((\eta, \omega) \in R^{2}, \gamma>0\right)$

$$
\begin{aligned}
& \lambda_{1}^{+}=\frac{-\tilde{\tau}}{w_{1}^{0}-\sigma}, \lambda_{2}^{ \pm}=\frac{\left(w_{1}^{0}-\sigma\right) \tilde{\tau} \mp c^{0} \sqrt{\tilde{\tau}^{2}-\omega^{2} d^{2}}}{d^{2}}, \\
& e_{1}^{+}={ }^{t}\left(\sqrt{2} \tilde{\tau}, \omega\left(w_{1}^{0}-\sigma\right),-\omega\left(w_{1}^{0}-\sigma\right)\right), \\
& e_{2}^{ \pm}={ }^{t}\left(-\omega \sqrt{2} c^{0}, \tilde{\tau}+\lambda_{2}^{ \pm}\left(w_{1}^{0}-\sigma-c^{0}\right), \tilde{\tau}+\lambda_{2}^{ \pm}\left(w_{1}^{0}-\sigma+c^{0}\right)\right),
\end{aligned}
$$

where $d^{2}=\left(c^{0}\right)^{2}-\left(w_{1}^{0}-\sigma\right)^{2}, \sqrt{1}=1$ and $\operatorname{Im} \lambda^{+}>0$ for $\gamma>0$. The determinant of the matrix:

$$
\left(\begin{array}{ccc}
\tilde{\tau}, & 0, & -\omega d \\
0, & 1, & -\beta
\end{array}\right)\left(e_{1}^{+}, e_{2}^{+}\right)
$$

is $\sqrt{2}\left(\tilde{\tau}+\lambda_{2}^{+}\left(r \omega_{1}^{0}-\sigma\right)\right)\left((1-\beta) \tilde{\tau}^{2}+\sqrt{2} \alpha\left(r \omega_{1}^{0}-\sigma\right) \omega^{2}+(1+\beta) \tilde{\tau} \sqrt{\tilde{\tau}^{2}-d^{2} \omega^{2}}\right)$. The vectors $e_{1}^{+}$and $e_{2}^{+}$are linearly independent if and only if $\tilde{\tau}+\lambda_{2}^{+}\left(w_{1}^{0}-\sigma\right) \neq 0$. From the above two fact, it follows that for $(\tau, \omega) \neq(0,0)$ the Lopatinskii determinant is always

$$
\begin{aligned}
& L=a_{1} \tilde{\tau}^{2}+a_{2} \omega^{2}-\tilde{\tau} \sqrt{\tilde{\tau}^{2}-d^{2} \omega^{2}}, \\
& a_{1}=-\frac{1-\beta}{1+\beta}<0, \quad a_{2}=-\frac{\sqrt{2} \alpha\left(w_{1}^{0}-\sigma\right)}{1+\beta}>0 .
\end{aligned}
$$

In [7], Majda shows that the conditions (1.2) and (1.3) for $\left\{U^{0 \pm}, \sigma\right\}$ imply the weak stability of the linearized problem (2.3) and (2.4) with respect to $\left\{U^{0^{+}}, \sigma\right\}$, i. e., $L(\tau, \omega) \neq 0$ for $\gamma>0$. Furthermore he prove that the above problem is uniformly stable, i. e., $L(\tau, \omega) \neq 0$ for $\gamma \geqq 0$, if and only if

$$
a_{1}+a_{2} \frac{1}{d^{2}}<0 \text {. }
$$


Under the above uniform stability, form his theorem it follows that for initial data described in section 1 for $l=1$ and sufficiently small $k_{0}^{\prime}$ there exists the shock front solution with the smoothness (1.4) for some $T$.

\section{Proof of Theorem.}

To show the sufficiency of the assertion of Theorem, we may only to prove the inequality (ii) of section 1 for the smooth shock front solution $\left\{U^{ \pm}, \beta\right\}$ with (1.4). Furthermore we may assume that $\left\{U^{ \pm}, \phi\right\}$ satisfy (2.1) and (2.2). Then we need only to estimate them. Note that the linearized problem with respect to $\left\{U^{0 \pm}, \sigma\right\}$ is assumed to be uniformly stable.

To obtain linear problem for $\left\{U^{ \pm}, \phi\right\}$, using (1.2) we rewrite (2.2) as follows :

$$
\begin{gathered}
\phi_{t}\left(F_{0}\left(U^{0+}+U^{\prime+}\right)-F_{0}\left(U^{0-}+U^{\prime-}\right)\right)+\phi_{x_{2}}\left(F_{2}\left(U^{0+}+U^{\prime+}\right)-F_{2}\left(U^{0-}+U^{\prime-}\right)\right) \\
+\left\{\sigma \int_{0}^{1} F_{0}^{\prime}\left(U^{0+}+\theta U^{\prime+}\right) d \theta-\int_{0}^{1} F_{1}^{\prime}\left(U^{0+}+\theta U^{++}\right) d \theta\right\} \cdot U^{+} \\
\quad-\left\{\sigma \int_{0}^{1} F_{0}^{\prime}\left(U^{0-}+\theta U^{\prime-}\right) d \theta-\int_{0}^{1} F_{1}^{\prime}\left(U^{0-}+\theta U^{\prime-}\right) d \theta\right\} \cdot U^{\prime-}=0 .
\end{gathered}
$$

Then regarding $A_{i}\left(U^{0 \pm}+U^{\prime \pm}\right), F_{i}\left(U^{0 \pm}+U^{\prime \pm}\right), \phi_{x_{i}} A_{i}$ and $\int_{0}^{1} F_{i}^{\prime} \cdot d \theta$ in (2.1) and (3.1) with fixed $\left\{U^{\prime \pm}, \phi\right\}$ as coefficients, and reflecting $U^{\prime-}$ with respect to $x_{1}=0$, we obtain a mixed problem for $U=\left\{U^{\prime+}, U^{\prime}\right\}$ and $\phi$ which is denoted by

$$
\begin{aligned}
& L^{+} U^{\prime+}=0, L^{-} U^{\prime-}=0 \quad \text { in }[0, T] \times\left\{x_{1}>0\right\} \text {, } \\
& B\left(U^{\prime+}, U^{-}, \phi\right)=0 \quad \text { on }[0, T] \times\left\{x_{1}=0\right\} \text {, } \\
& U^{\prime+}(0, x)=h^{+}(x) \text { and } U^{\prime-}(0, x)=h^{-}(x) \text {. }
\end{aligned}
$$

We may choose $T$ and $k_{0}$ in (1.4) sufficiently small numbers such that $\left\{L^{ \pm}\right\}$in (3.2) is strongly hyperbolic and the linear mixed problem (3.2) is also uniformly stable in Kreiss-Majda's sense. We let $U_{1}=\left\{U_{1}^{+}, U_{1}^{-}\right\}$and $\phi_{1}$ be the solution of (3.2) with zero initial data and with $B\left(U_{1}^{+}, U_{1}^{-}, \phi_{1}\right)=g$ for some $g \in L^{2}\left(t>0, x_{1}=0\right)$. We obtain the estimate for $U_{1}$ and $\phi_{1}$ :

$$
\begin{aligned}
& \left\|U_{1}\right\|_{0,(0, T) \times\left\{x_{1}>0\right\}}^{2}+\left\langle\left\langle U_{1}\right\rangle_{0,(0, T) \times\left\{x_{1}=0\right\}}^{2}+\left\langle\left\langle\phi_{1}\right\rangle_{0,(0, T) \times\left\{x_{1}=0\right\}}^{2}\right.\right. \\
& \quad \leqq c_{1}\left\langle\langle g\rangle_{0,(0, T) \times\left\{x_{1}=0\right\}}^{2} .\right.
\end{aligned}
$$

We let also $U_{2}=\left(U_{2}^{+}, U_{2}^{-}\right)$be the solution of the problem :

$$
\begin{aligned}
& L^{+} U_{2}^{+}=0, L^{-} U_{2}^{-}=0 \quad \text { in }[0, T] \times\left\{x_{1}>0\right\}, \\
& N^{+} U_{2}^{+}=0, N^{-} U_{2}^{-}=0 \quad \text { in }[0, T] \times\left\{x_{1}=0\right\}, \\
& U_{2}^{+}(0, x)=h^{+}(x) \text { and } \quad U_{2}^{-}(0, x)=h^{-}(x),
\end{aligned}
$$


where $N^{+}(x)$ is the projection of the subspace in $C^{3}$ spaned by positive eigenvectors of the coefficient of $\frac{\partial}{\partial x_{1}}$ in $L^{+}$. Then since $L^{ \pm}$is symmetric, by Rauch's method ([11]) we obtain the desired estimate corresponding to (ii). That is, by the Energy method it follows that

$$
\begin{aligned}
& \left\|U_{2}(t, x)\right\|_{0,\left\{x_{1}>0\right\}}^{2}+\left\langle\left\langle U_{2}\right\rangle\right\rangle_{0,(0, T) \times\left\{x_{1}=0\right\}}^{2} \\
& \quad \leqq c_{2}\|h\|_{0,\left\{x_{1}>0\right\}}^{2} \quad \text { for } \quad t \in[0, T] .
\end{aligned}
$$

Thus setting $g=-B\left(U_{2}^{+}, U_{2}^{-}, 0\right)$ and $\tilde{U}^{\prime}=U_{1}+U_{2}$, by the uniqueness theorem of the linear problem we obtain that $\tilde{U}^{\prime}=U^{\prime}, \phi_{1}=\phi$ and the desired estimate for $\left\{U^{\prime}, \phi\right\}$. For, the symmetricity of $L^{ \pm}$yields the estimate : for $t \in[0, T]$.

$$
\left\|U_{1}(t, x)\right\|_{0,\left\{x_{1}>0\right\}}^{2} \leqq c_{3}\left\langle\left\langle U_{1}\right\rangle\right\rangle_{0,0, T) \times\left\{x_{1}=0\right\}}^{2} .
$$

Here we remark also that the uniqueness of solutions with (1.4) is derived as above by using a linear equations satisfied by the difference of solutions.

Next, to prove the necessity of the assertion of Theorem, we assume that (i) and (ii) are valid. Now we shall show that the linearized problem (2.3) and (2.4) is $L^{2}$-well posed in our sense ([1]). We let $\left\{U^{\prime \pm}, \phi\right\}$ be functions such that their derivatives satisfy the conditions with respect to initial data in section $1,\left\{U^{\prime \pm}, \phi\right\} \neq 0, \operatorname{Supp}\left(U^{\prime+}\right) \cap\left\{x_{1} \leqq \delta\right\}=\emptyset$ for a fixed $\delta \ll 1$ and $U^{\prime-} \equiv 0$. We let $\left\{U^{0 \pm}+U_{c}^{\prime \pm}, \sigma t+\phi_{s}\right\}$ be the solution with the initial data $\left\{U^{0+}+\varepsilon U^{+}, U^{0-}, \sigma t+\varepsilon \phi\right\}$ with $\varepsilon>0$, whose existence is assumed in (i). Then $U_{c}^{\prime-}=0$ and $\left(U_{c}^{\prime+}, \phi_{s}\right\}$ is a solution to the problem $(2.1)^{+}$and (3.1), for which (1. 4) and (ii) are valid. Let $h(t)$ be a cut off function such that $h(t) \in C^{\infty}(R)$, $h(t)=1$ for $t \leqq T-\delta$ and $h(t)=0$ for $t \geqq T$. Here we note that $\phi$ and $U^{\prime+}$ vanish on $\left\{(t, x) \mid t=0,0<x_{1}<\delta\right\}$.

We let $\left\{V_{s}, \phi_{s}\right\}$ be

$$
\begin{aligned}
& V_{c}=h \cdot \frac{1}{\varepsilon} U_{c}^{+\prime}, \\
& \phi_{c}=h \cdot \frac{1}{\varepsilon} \phi_{s} \quad \text { for } \quad t \geqq 0 .
\end{aligned}
$$

Then from (ii) it follows that for some $c_{1}>0$

$$
\left\|V_{\star}\right\|_{0,\left\{t>0, x_{1}>0\right\}} \leq c T\left\|V_{\imath}\right\|_{0,\left\{t=0, x_{1}>0\right\}} \leqq c_{1} .
$$

We have from $(2.1)^{+}$and (3.1) that

$$
\begin{aligned}
& L_{\varepsilon}\left(V_{\varepsilon}\right) \\
& \quad \equiv A_{0}\left(U^{0+}+\varepsilon V_{\varepsilon}\right) \frac{\partial V_{\varepsilon}}{\partial t}+\left(A_{1}\left(U^{0+}+\varepsilon V_{\varepsilon}\right)-\sigma A_{0}\left(U^{0+}+\varepsilon V_{\diamond}\right)\right) \frac{\partial V_{\varepsilon}}{\partial x_{1}}
\end{aligned}
$$




$$
\begin{aligned}
& +A_{2}\left(U^{0+}+\varepsilon V_{\varepsilon}\right) \frac{\partial V_{\varepsilon}}{\partial x_{2}}-\left\{\varepsilon \psi_{\varepsilon}, A_{0}\left(U^{0+}+\varepsilon V_{\varepsilon}\right)\right. \\
& \left.+\varepsilon \psi_{\varepsilon, x_{2}} A_{2}\left(U^{0+}+\varepsilon V_{s}\right)\right\} \frac{\partial V_{\varepsilon}}{\partial x_{1}} \\
& =\frac{1}{\varepsilon} f_{\mathrm{c}} \quad \text { in } \quad\left\{(t, x) \mid t \geqq 0, x_{1} \geqq 0\right\} \text {, } \\
& \phi_{\iota, t}\left(F_{0}\left(U^{0+}+\varepsilon V_{\imath}\right)-F_{0}\left(U^{0-}\right)\right)+\phi_{s, x_{2}}\left(F_{2}\left(U^{0+}+\varepsilon V_{s}\right)-F_{2}\left(U^{0-}\right)\right) \\
& +\left\{\sigma \int_{0}^{1} F_{0}^{\prime}\left(U^{0+}+\theta \varepsilon V_{\varepsilon}\right) d \theta-\int_{0}^{1} F_{1}^{\prime}\left(U^{0+}+\theta \varepsilon V_{\imath}\right) d \theta\right\} V_{\iota} \\
& =0 \quad \text { on } \quad\left\{(t, x) \mid 0 \leqq t \leqq T-\delta, x_{1}=0\right\} \text {, }
\end{aligned}
$$

where $\operatorname{Supp}\left(f_{\mathrm{c}}\right) \subset\left\{T-\delta<t<T, x_{1} \geqq 0\right\}$. By the usual calculations with respect to Sobolev norms we see that $f_{\mathrm{d}} \mid\{t \geq 0\}$ is a sum of terms containg $U_{*}^{\prime+}$ with bounded factors, and hence that $\left\|\frac{1}{\varepsilon} f_{s}\right\|_{0,\{t \geq 0\}} \leqq c_{2}$ for some constant $c_{2}$. Now, we use the estimate

$$
\begin{aligned}
& \langle U\rangle\rangle_{-\frac{1}{2},\left\{x_{1}=0\right\}} \leqq c_{3}\|U\|_{1,-1,\left\{x_{1}>0\right\}} \\
& \quad \leqq c_{4}\left(\left\|L_{\varepsilon}(U)\right\|_{0,\left\{x_{1}>0\right\}}+\|U\|_{0,\left\{x_{1}>0\right\}}\right),
\end{aligned}
$$

where $U \in H^{1}\left(\left\{x_{1}>0\right\}\right)$. ([4]). We set $\chi$ a cut off function $\in C_{0}^{\infty}\left(R^{\prime}\right)$ such that $\chi\left(x_{1}\right)=1$ for $x_{1}<\delta / 2$ and $\chi\left(x_{1}\right)=0$ for $x_{1}>\delta$. Then from the above estimate we have:

$$
\begin{aligned}
& \left\langle V_{\imath}\right\rangle_{-\frac{1}{2},\left\{x_{1}=0\right\}} \\
& \quad \leqq c_{4}\left(\left\|L_{\imath} \chi V_{\bullet}\right\|\left\|_{0,\left\{x_{1}>0\right\}}+\right\| \chi V_{\imath} \|_{0,\left\{x_{1}>0\right\}}\right) \\
& \quad \leqq c_{5}\left(\left\|L_{\imath} V_{\imath}\right\|_{0,\left\{t>0, x_{1}>0\right\}}+\left\|V_{\triangleleft}\right\|_{0,\left\{t>0, x_{1}>0\right\}}\right) \\
& \quad \leq c_{6},
\end{aligned}
$$

where $c_{6}$ also dependent on $\chi^{\prime}$ and hence only on $\delta, T c|| V_{8} \|_{0,\left\{t=0, x_{1} \geq 0\right\}}$ and $k_{0}$, but not on $\varepsilon$. Furthermore for sufficiently small $k_{0},(1.2)$ and (1.3) imply that $F_{0}\left(U^{0+}+\varepsilon V_{\varepsilon}\right)-F_{0}\left(U^{0-}\right)$ and $F_{2}\left(U^{0+}+\varepsilon V_{\varepsilon}\right)-F_{2}\left(U^{0-}\right)$ are linearly independent and hence (3.4) yields that $\phi_{c, t}$ and $\phi_{c, x_{2}}$ are represented as linear combinations of elements of $V_{\varepsilon}$ with coefficients in $H^{s-\frac{1}{2}}\left(\left\{t<T-\delta, x_{1}=0\right\}\right)$ Using the boundedness of $V$, and the above fact, there exist a weak limit $\{V, \phi\}$ such that

$$
V_{\mathrm{e}^{\prime}} \underset{w}{\longrightarrow} V \quad \text { in } \quad H^{0}\left(\left\{0<t<T, x_{1}>0\right\}\right),
$$




$$
V_{s^{\prime}} \underset{w}{\longrightarrow} V \quad \text { in } H^{-\frac{1}{2}}\left(\left\{x_{1}=0\right\}\right),
$$

$$
\begin{array}{lll}
\varepsilon^{\prime} V_{s^{\prime}} \longrightarrow 0 & \text { in } \quad H^{s-1}\left(\left\{0<t<T, x_{1}>0\right\}\right), \\
V_{\iota^{\prime}}=U^{\prime+}(0, x) & \text { in } \quad H^{s}\left(\left\{t=0, x_{1}>0\right\}\right) \quad \text { and } \\
\phi_{\iota^{\prime}} \underset{w}{\longrightarrow} \phi & \text { in } \quad H^{\frac{1}{2}}\left(\left\{t<T-\delta, x_{1}=0\right\}\right)
\end{array}
$$

as $\varepsilon^{\prime} \rightarrow 0$. Here we note that $\operatorname{Supp}_{x_{2}}\left(\phi_{\varepsilon^{\prime}}\right) \subset\left\{\left|x_{2}\right| \leqq 1\right\}$.

Accordingly for any $W \in C_{0}^{\infty}\left(\left\{0<\mathrm{t}<\mathrm{T}-\delta, 0<x_{1}<\infty\right\}\right)$

$$
\int\left\langle\left(A_{0}\left(U^{0+}+\varepsilon^{\prime} V_{\varepsilon^{\prime}}^{+}\right)-A_{0}\left(U^{0+}\right)\right) \frac{\partial V_{t^{\prime}}^{+}}{\partial t}, W\right\rangle d t d x \rightarrow 0
$$

as $\varepsilon^{\prime} \rightarrow 0$. Because then

$$
A_{0}\left(U^{0+}+\varepsilon^{\prime} V_{\varepsilon^{\prime}}^{+}\right)-A_{0}\left(U^{0+}\right) \longrightarrow 0
$$

in $H^{s-1}\left(\left\{0<t<T, x_{1}>0\right\}\right)$.

Therefore we have that $V$ is a weak solution of the linearized problem $\left(2.1^{\prime}\right)^{+}$and $\left(2.2^{\prime}\right)$ with initial data $V=U^{\prime+}(0, x) \in H_{0}^{s+1}\left(\left\{x_{1}>\delta\right\}\right)$ and (3.5) such that

$$
\begin{aligned}
& \|V(t, x)\|_{0,\left\{0,<t<T-\delta, x_{1}>0\right\}} \leqq c T\left\|U^{+}(0, x)\right\|_{0,\left\{x_{1}>0\right\}}, \\
& \quad \int\left\langle L_{0}^{+}\left(V_{\iota^{\prime}}\right), W\right\rangle d t d x=-\int\left\langle V_{\iota^{\prime}}, L_{0}^{+} W\right\rangle d t d x \rightarrow 0, \\
& \quad \int\left\langle\mathrm{B}_{0}\left(V_{\mathrm{e}^{\prime}}, \psi_{\mathrm{\iota}^{\prime}}\right), W_{1}\right\rangle d t d x_{2} \rightarrow 0
\end{aligned}
$$

as $\varepsilon^{\prime} \rightarrow 0$.

Here $L_{0}^{+}, B_{0}$ are the linearized operators in $\left(2.1^{\prime}\right)^{+}$and $\left(2.2^{\prime}\right)$ respectively and $W_{1} \in C_{0}^{\infty}\left(\left\{0 \leqq t<T-\delta, x_{1}=0\right\}\right)$.

On the other hand, the weak stability of the linear problem (2.3) and (2.4) implies that for initial data $N_{0}^{-1} U^{\prime+}(0, x)$, as it is shown below in section 3 , there exist a unique solution $N_{0}^{-1} V_{1} \in H^{s-1}\left(\left\{0<t<T-\delta, x_{1}>0\right\}\right)$ and hence from (2.5) there is a unique solution $\left\{V_{1}, \phi_{1}\right\}$ of the problem $\left(2.1^{\prime}\right)^{+},\left(2.2^{\prime}\right)$. Therefore we have that $\left\{V_{\varepsilon^{\prime}}-V_{1}, \phi_{\varepsilon},-\phi_{1}\right\}$ satisfies (3.5) replaced $V$, $U^{\prime+}$ $(0, x)$ and $\phi$ by $V-V_{1}, 0$ and $\phi-\phi_{1}$ respectively. Furthermore we have, as $\varepsilon^{\prime} \rightarrow 0$

$$
\begin{aligned}
& \int\left\langle V_{s^{\prime}}-V_{1}, L_{0}^{+} \widetilde{W}\right\rangle d t d x \rightarrow 0, \\
& \int\left\langle B_{0}\left(V_{\iota^{\prime}}-V_{1}, \phi_{s^{\prime}}-\phi_{1}\right), W_{1}\right\rangle d t d x_{2} \rightarrow 0,
\end{aligned}
$$


where $\widetilde{W} \in C_{0}^{\infty}\left(\left\{0 \leqq t<T-\delta, 0<x_{1}<\infty\right\}\right)$. Hence considering the convolution by a function $q_{n}\left(t, x_{2}\right)=n^{2} g\left(n t, n x_{2}\right) \in C_{0}^{\infty}\left(R^{2}\right)$, we see that

$$
\begin{array}{ll}
L_{0}^{+}\left(q_{n} *\left(V-V_{1}\right)\right)=0 & \text { for } t \leqq T-2 \delta, x_{1}>0, \\
B_{0}\left(q_{n} *\left(V-V_{1}\right), q_{n} *\left(\phi-\phi_{q}\right)\right)=0 & \text { for } t \leqq T-2 \delta, \\
q_{n} *\left(V-V_{1}\right)=q_{n} *\left(\phi-\phi_{1}\right)=0 & \text { for } t \leqq-\frac{1}{n} .
\end{array}
$$

These equalities imply that $N_{0}^{-1} q_{n} *\left(V-V_{1}\right)$ is a solution of (2.3) and (2.4) for $t \leqq T-2 \delta$ with zero initial data at $t=-\frac{1}{n}$.

By the uniqueness of the smooth solution of the above problem, we have $N_{0}^{-1} V \in H^{s-1}\left(\left\{0<t<T-2 \delta, x_{1}>0\right\}\right)$. Thus we see that there exists the solution of (2.3) and (2.4) $N_{0}^{-1} V \in H^{s-1}\left(\left\{0<t<T-2 \delta, x_{1}>0\right\}\right)$ such that $\left\|N_{0}^{-1} V(t, x)\right\|_{0,\left\{0<t<T-2 \delta, x_{1}>0\right\}} \leq c T\left\|N_{0}^{-1} U^{\prime+}(0, x)\right\|_{0,\left\{x_{1} \geq 0\right\}}$.

Obviously for the above inequality the restrictions with respect to norms of initial data are removed. Regarding the pure Cauchy problem and the partition of unity we can also remove the condition that support of data $\subset\left\{|x| \leqq \frac{1}{2}\right\}$.

Extending $V$, and $\varepsilon V$, suitably to $\{t<0\}$, by the same way as above we can also remove the condition that (support of data) $\cap\left\{x_{1} \leq \delta\right\}=\emptyset$. Thus by Duhamel's principle we obtain that for all $F(t, x) \in C^{1}\left([0, T-2 \delta], H_{0}^{s+l}\right.$ $\left.\left(\left\{x_{1}>0\right\}\right)\right)$ the solution $U(t, x)$ of $(2.3)$ with the right hand side $F$ and (2.4) with zero initial data satisfies: for some $c_{0}>0$

$$
\|U(t, x)\|_{0,\left\{0<t<T-2 \delta, x_{1}>0\right\}} \leqq c_{0}\|F(t, x)\|_{0,\left\{0<t<T-2 \delta, x_{1}>0\right\}},
$$

which is just the $L^{2}$-well posedness of the linearized problem (2.3) and (2.4). For, by limit process, we can weaken the condition of $F$ above to that $F \in H_{0}^{1}\left(\left\{0<t<T-2 \delta, 0<x_{1}\right\}\right)$. The proof of the assertion of Theorem is complete, provided the above $L^{2}$-well posedness yields the uniform stability of the same problem. Its proof is given in the next section.

\section{Reflection coefflcients.}

In this section we study the linearized problem (2.3) and (2.4) with respect to the step shock front satisfying (1.2) and (1.3).

We let $N$ be the matrix :

$$
N=\left(e_{1}^{+}, e_{2}^{+}, e_{2}^{-}\right),
$$


whose components are functions of $(\tilde{\tau}, \omega)$ for fixed $\left\{U^{0 \pm}, \sigma\right\}$. Hereafter we consider only statements with respect to $(\tau, \omega)$ which is normalized and has small imaginary part $\gamma$ of $\tau$. Then as stated in section $2 e_{1}^{+}$and $e_{2}^{+}$are linearly independent and the three vectors are also linearly independent whenever $\gamma \neq 0$. Assuming $\gamma>0$ we let $\hat{U}={ }^{t}\left(U_{1}, U_{2}, U_{3}\right)$ be the vector such that for a solution $V(t, x)$ of (2.3) vanishing for $t<0$

$$
\hat{U}=N^{-1} \hat{V} \text {. }
$$

Then $\hat{U}$ satisfies the following relations :

$$
\begin{aligned}
& D_{x} \hat{U}=\left(\begin{array}{ccc}
\lambda_{1}^{+} & & \\
& \lambda_{2}^{+} & \\
& & \\
& \lambda_{2}^{-}
\end{array}\right) \hat{U} \quad \text { in }\left\{x_{1} \geqq 0\right\}, \\
& \hat{B}\left(e_{1}^{+}, e_{2}^{+}\right)^{t}\left(U_{1}, U_{2}\right)+\hat{B}\left(e_{2}^{-}\right) U_{3}=\hat{B} \hat{V} \quad \text { on }\left\{x_{1}=0\right\} .
\end{aligned}
$$

By assumption of the weak stability of the linearized problem we have:

$$
\begin{aligned}
& \left|\hat{B}\left(e_{1}^{+}, e_{2}^{+}\right)\right| \neq 0 \quad \text { for } \quad \gamma>0, \\
& { }^{t}\left(U_{1}, U_{2}\right)+\hat{B}\left(e_{1}^{+}, e_{2}^{+}\right)^{-1} \cdot \hat{B}\left(e_{2}^{-}\right) U_{3}=\hat{B}\left(e_{1}^{+}, e_{2}^{+}\right)^{-1} \hat{B} \hat{V},
\end{aligned}
$$

where

$$
\left(\hat{B}\left(e_{1}^{+}, e_{2}^{+}\right)\right)^{-1} \cdot \hat{B}\left(e_{2}^{-}\right) \equiv{ }^{t}\left(\widetilde{b_{12}}, \widetilde{b_{22}}\right)
$$

is called by reflection coefficients with respect to our problem.

We already have that for our problem is $L^{2}$-well posed if and only if for some $c \geq 0$

$$
\begin{aligned}
& \left|\widetilde{b_{12}}\right| \leqq c \gamma^{-1}\left|\operatorname{Im} \lambda_{1}^{+}\right|^{\frac{1}{2}}\left|\operatorname{Im} \lambda_{2}^{-}\right|^{\frac{1}{2}}\left|\lambda_{2}^{-}-\lambda_{2}^{+}\right|, \\
& \left|\widetilde{b_{22}}\right| \leqq c \gamma^{-1}\left|\operatorname{Im} \lambda_{2}^{+}\right|^{\frac{1}{2}}\left|\operatorname{Im} \lambda_{2}^{-}\right|^{\frac{1}{2}}\left|\lambda_{2}^{-}-\lambda_{2}^{+}\right|,
\end{aligned}
$$

whenever Lopatinskii determinant $\left|\hat{B}\left(e_{1}^{+}, e_{2}^{+}\right)\right|\left(\tau^{0}, \omega^{0}\right)=0$. Here $\tau^{0}$ is real and $(\tau, \omega)$ are in a neighborhood of $\left(\tau^{0}, \omega^{0}\right)$ with $\gamma>0$. ([10], [12]).

By direct calculations yield :

$$
\begin{aligned}
& \widetilde{b_{12}} \times\left|\hat{B}\left(e_{1}^{+}, e_{2}^{+}\right)\right|=\left|\hat{B}\left(e_{2}^{-}\right), \hat{B}\left(e_{2}^{+}\right)\right| \\
& \quad=\tilde{\tau}\left(\lambda_{2}^{+}-\lambda_{2}^{-}\right) \omega \sqrt{2} c^{0}\left\{\sqrt{2} \alpha+(\beta+1) c^{0}+(\beta-1)\left(w_{1}^{0}-\sigma\right)\right\}, \\
& \widetilde{b_{22}} \times\left|\hat{B}\left(e_{1}^{+}, e_{2}^{+}\right)\right|=\left|\hat{B}\left(e_{1}^{+}\right), \hat{B}\left(e_{2}^{-}\right)\right| \\
& \quad=\left\{\tilde{\tau}+\lambda_{2}^{-}\left(w_{1}^{0}-\sigma\right)\right)\left\{(1-\beta) \tilde{\tau}^{2}+\sqrt{2} \alpha\left(w_{1}^{0}-\sigma\right) \omega^{2}-(1+\beta) \sqrt{\tilde{\tau}^{2}-d^{2} \omega^{2}} \tilde{\tau}\right\},
\end{aligned}
$$

where $\operatorname{Im}(-\sqrt{7})>0$ for $\gamma>0$. 
Here we remark that if the stability function $a_{1}+a_{2} d^{-2}>0$, the zeros of Lopatinskii determinant occur at $(\eta, \omega)$ contained in the projection to the hyperplane $\{\lambda=0\}$ of the interior of the normal cone. In this case the problem is not $L^{2}$-well posed. ([12]). Therefore we need only to treat the case where $a_{1}+a_{2} d^{-2}=0$. But for the case where $a_{1}+a_{2} d^{-2} \geqq 0$ we examine the vanishing order of reflection coefficients in order to clarify the degnerate order of well posedness of our problem.

To do so, we first show that the following two functions are equivalent in a neighborhood of $\left(\tilde{\tau}_{0}, \omega_{0}\right)$ i. e.,

$$
\widetilde{b_{12}}\left|\hat{B}\left(e_{1}^{+}, e_{2}^{+}\right)\right| \sim\left|\lambda_{2}^{+}-\lambda_{2}^{-}\right| \text {. }
$$

we let $(\tilde{\tau}, \omega)$ belong to a neighborhood of $\left(\tilde{\tau}_{0}, \omega_{0}\right)$ for which $L\left(\tilde{\tau}_{0}, \omega_{0}\right)=0$. If $\tilde{\tau}_{0}=0,(2.6)$ implies $L\left(\tilde{\tau}_{0}, \omega_{0}\right)=-a_{2} \omega_{0}^{2}$ and hence $\omega_{0}=0$, which is contrary to our assumption $\left(\tau_{0}, \omega_{0}\right) \neq 0$. By the same way we see that $\omega_{0} \neq 0$ and we need only to show:

$$
\sqrt{2} \alpha+(\beta+1) c^{0}+(\beta-1)\left(w_{1}^{0}-\sigma\right) \neq 0 .
$$

We divide this by $\beta+1>0$, then from (2.6) it becomes

$$
-a_{2}\left(w_{1}^{0}-\sigma\right)^{-1}+c^{0}+a_{1}\left(w_{1}^{0}-\sigma\right) .
$$

Since we can rewrite $a_{1}$ and $a_{2}$ as follows :

$$
\begin{aligned}
& a_{1}=-\frac{1}{2}\left\{\left(c^{0}\right)^{2}+\left(w_{1}^{0}-\sigma\right)^{2}\right\}\left(c^{0}\right)^{-1}\left(w_{1}^{0}-\sigma\right)^{-1}, \\
& a_{2}=\frac{1}{2}[P]\left([\rho] c^{0}\right)^{-1} d^{2}\left(w_{1}^{0}-\sigma\right)^{-1},
\end{aligned}
$$

where $[\rho]=\rho^{0+}-\rho^{0-}$, we need only to show that

$$
\left(c^{0}\right) \neq \frac{1}{2}\left\{\frac{\left(c^{0}\right)^{2}+\left(w_{1}^{0}-\sigma\right)^{2}}{c^{0}}+\frac{[P]}{[\rho] c^{0}} \frac{\left(c^{0}\right)^{2}-\left(w_{1}^{0}-\sigma\right)^{2}}{\left(w_{1}^{0}-\sigma\right)^{2}}\right\} .
$$

Using the fact that

$$
\frac{[P]}{[\rho]}=\frac{\rho^{+0}}{\rho^{-0}}\left(w_{1}^{0}-\sigma\right)^{2},
$$

we reduce our problem to show:

$$
\left(c^{0}\right)^{2} \neq \frac{1}{2}\left(1+\frac{\rho^{0+}}{\rho^{0-}}\right)\left(c^{0}\right)^{2}+\frac{1}{2}\left(1-\frac{\rho^{0+}}{\rho^{0-}}\right)\left(w_{1}^{0}-\sigma\right)^{2} .
$$

From (1.2) and (1.3) it implies that $\rho^{0+} \neq \rho^{0-}$ and by (1.3) we have that $\left(c^{0}\right)^{2}>\left(w_{1}^{0}-\sigma\right)^{2}$. Hence the above inequality is valid. 
Next we remark that

$$
\tilde{\tau}+\lambda_{2}^{-}\left(w_{1}^{0}-\sigma\right) \neq 0 .
$$

For, if $\tilde{\tau}+\lambda_{2}^{-}\left(w_{1}^{0}-\sigma\right)=0$, then from the relation $\left(c^{0}\right)^{2}=d^{2}+\left(w_{1}^{0}-\sigma\right)^{2}$ it follows that $\tilde{\tau}=-i|\omega|\left(w_{1}^{0}-\sigma\right)$. But $\operatorname{Im} \lambda_{2}^{-}<0$ and hence $\tilde{\tau}+\lambda_{2}^{-}\left(w_{1}^{0}-\sigma\right) \neq 0$, which is a contradiction. Thus we have that

$$
\widetilde{b_{22}} \cdot\left|\tilde{B}\left(e_{1}^{+}, e_{2}^{+}\right)\right| \sim a_{1} \tilde{\tau}^{2}+a_{2} \omega^{2}+\tilde{\tau} \sqrt{\tilde{\tau}^{2}-d^{2} \omega^{2}} .
$$

Let $a_{1}+a_{2} d^{-2}=0$, then for the point $\left(\tilde{\tau}_{0}, \omega_{0}\right)$ such that $\tilde{\tau}_{0}^{2}=d^{2} \omega_{0}^{2}$ Lopatinskii determinant $L\left(\tau_{0}, \omega_{0}\right)=0$. We let $\tilde{\tau} \pm d \omega_{0}=\zeta$ for $\tilde{\tau}_{0}=\mp d \omega_{0}$ respectively. Then from (4.4) and (4.5) it follows that

$$
\begin{aligned}
& \left|\widetilde{b_{12}}\right| \sim \frac{k_{0} \sqrt{\zeta}}{k_{1} \sqrt{\zeta}+k_{2} \zeta}, \\
& \left|\widetilde{b_{22}}\right| \sim \frac{k_{1} \sqrt{\zeta}+k_{2}^{\prime} \zeta}{k_{1} \sqrt{\zeta}+k_{2} \zeta},
\end{aligned}
$$

where $k_{0}$ and $k_{1} \neq 0$. Therefore we have that both of $\left|\widetilde{b_{12}}\right|$ and $\left|\widetilde{b_{22}}\right|$ are bounded and do not vanish. Since $\left|\operatorname{Im} \lambda_{1}^{+}\right| \sim \gamma, \gamma \sim(\operatorname{Re} \sqrt{\zeta}) \cdot(\operatorname{Im} \sqrt{\zeta})$ and $\lambda_{2}^{-}-$ $\lambda_{2}^{+} \sim \sqrt{\zeta}$, we see that the first term of the right hand side in (4.2) $\sim \sqrt{\zeta}(\operatorname{Re} \sqrt{\zeta})^{-\frac{1}{2}}$, which tends to zero when $\zeta=-i \gamma$. Furthermore the second term $\sim \sqrt{\zeta}(\operatorname{Re} \sqrt{\zeta})^{-1}$, which is bounded from below by positive constant. Thus we have that $(4.2)$ are valid for $\widetilde{b_{22}}$, but not for $\widetilde{b_{12}}$.

Next, we let $a_{1}+a_{2} d^{-2}>0$. Then also from (4.4), (4.5) and the simple multiplicity of the zeros of $L$ it follows that both of $\widetilde{b_{12}}$ and $\widetilde{b_{22}}$ are $0\left(\gamma^{-1}\right)$. But in this case terms of the right hand side of (4.2) are bounded, since both of $\lambda_{1}^{+}$and $\lambda_{2}^{+}$are simple. Hence we see that (4.2) are not valid and the problem is $L^{2}$-well posed if and only if it is uniformly stable.

Finally we remark that for some $c>0$

$$
\begin{aligned}
& \left|\widetilde{b_{12}}\right| \leqq c \gamma^{-2}\left|\operatorname{Im} \lambda_{1}^{+}\right|^{\frac{1}{2}}\left|\operatorname{Im} \lambda_{2}^{+}\right|^{\frac{1}{2}}\left|\lambda_{2}^{-}-\lambda_{2}^{+}\right|, \\
& \left|\widetilde{b_{22}}\right| \leqq c \gamma^{-2}\left|\operatorname{Im} \lambda_{2}^{+}\right|^{\frac{1}{2}}\left|\operatorname{Im} \lambda_{2}^{-}\right|^{\frac{1}{2}}\left|\lambda_{2}^{-}-\lambda_{2}^{+}\right|
\end{aligned}
$$

even if $a_{1}+a_{2} d^{-2} \geqq 0$. Therefore the linearized problem is always $L^{2}$-well posed with decreasing order 1 . Note that the plane $\left\{x_{1}=0\right\}$ is non-characteristic for operators (2.3) and (2.4). Hence for a given vector $F(t, x) \in$ $H_{0}^{s+1}\left(\left\{t>0, x_{1}>0\right\}\right)$. there exists a unique solution $U$ to problem (2.3) with the right hand side $F$ and (2.4) with homogeneous boundary conditions such that $\operatorname{supp}(U) \cap\{t<0\}=\emptyset$, and for some $c_{T}$

$$
\|U\|_{s,\left\{0<t<T, x_{1}>0\right\}} \leqq c_{T}\|F\|_{s+1,\left\{0<t<T, x_{1}>0\right\}} .
$$


([1], [10]). Therefore we have : for a given initial data $U(0, x) \in H_{0}^{s+1}\left(\left\{x_{1}>0\right\}\right)$ there is a unique solution $U(t, x) \in H^{s-1}\left(\left\{t>0, x_{1}>0\right\}\right)$ to the problem (2.3) and (2.4) such that

$$
\|U\|_{s-1,\left\{0<t<T, x_{1}>0\right\}} \leqq c_{T}\|U(0, x)\|_{s+1,\left\{x_{1}>0\right\}} .
$$

\section{Conclusion and an example.}

1) From Theorem we have that if the linearized problem with respect to $\left\{U^{0 \pm}, \sigma\right\}$ is only weakly stable, the stability condition (ii) is not satisfied for any short time, even if (i) is valid until some time. Though here we deal mainly with piecewise smooth shock front solutions, this fact will correspond to the instability in Fluid mechanics, ([5], [14]). However, it is not known even whether there exists an other such shock front solution with the same initial data as $\left\{U^{0 \pm}, \sigma t\right\}$, when the linearized problem with respect to $\left\{U^{0 \pm}, \sigma\right\}$ is only weakly stable.

The same treatment as above is applicable to 3-dimensional problems.

2) Example.

The stability of the linearized problem with respect to $\left\{U^{0 \pm}, \sigma\right\}$ depends only on $\left\{\rho^{0 \pm}\right\}$ under the conditions (1.2) and (1.3). That is, for 1-shock front it is uniformly stable if and only if

$$
P\left(\rho^{0+}\right)-P\left(\rho^{0-}\right)<P_{\rho}\left(\rho^{0+}\right) \rho^{0+} .
$$

([7]).

We let

$$
P(\rho)=1+\left(1-\frac{1}{\rho}\right)(1+\varepsilon(\rho-1))^{2} \quad \text { for } \quad \rho>1 \text { and }
$$

for some positive $\varepsilon \ll 1$. $([9])$.

Since $P_{\rho}=(1+\varepsilon(\rho-1)) \rho^{-2}\{1+\varepsilon(\rho-1)(1+2 \rho)\}$ and

$$
P_{\rho \rho}=2 \rho^{-3}\left(\rho^{3} \varepsilon^{2}-(1-\varepsilon)^{2}\right),
$$

setting $\rho_{0}=\left((1-\varepsilon) \varepsilon^{-1}\right)^{\frac{2}{3}}$, we have that

$$
\begin{aligned}
& P_{\rho}>0 \quad \text { for } \rho>1, P_{\rho \rho}<0 \quad \text { for } \rho \in\left(1, \rho_{0}\right), \\
& P_{\rho \rho}\left(\rho_{0}\right)=0 \text { and } P_{\rho \rho}>0 \text { for } \rho>\rho_{0} .
\end{aligned}
$$

If $\rho^{+}>\rho^{-}$, for any $w_{1}^{+}$and $w_{2}^{+}=w_{2}^{-}$we can find $w_{1}^{-}$and $\sigma$ such that $U^{+}={ }^{t}\left(w_{1}^{+}, w_{2}^{+}, P\left(\rho^{+}\right)\right), U^{-}={ }^{t}\left(w_{1}^{-}, w_{2}^{-}, P\left(\rho^{-}\right)\right)$and $\sigma$ satisfy (1.2) and (1.3). In fact the 1 -shock is genuinely nonlinear. Now the maximum of $P(\rho)-P_{\rho}(\rho) \cdot \rho$ occus at $\rho_{0}$. We see that for sufficiently small $\varepsilon$ 


$$
P\left(\rho_{0}\right)-P_{\rho}\left(\rho_{0}\right) \cdot \rho_{0}>1
$$

We let $\rho^{0+}=\rho^{0}$. Then there exists $\rho_{1}$ such that $1<\rho_{1}<\rho_{0}$, the linearized problem is uniformly stable for $\left\{\rho^{0+}, \rho^{0-}\right\}$ provided : $\rho^{0-} \in\left(\rho_{1}, \rho_{0}\right)$, and is only weakly stable for $\left\{\rho^{0+}, \rho^{0-}\right\}$ provided : $1<\rho^{0-} \leqq \rho_{1}$.

\section{References}

[1] R. Agemi-T. Shirota: On necessary and sufficient conditions for $L^{2}$-wellposedness of mixed problems for hyperbolic equations I, II, J. Fac. Sci. Hokkaido Univ., Vol. 21, No. 2, 133-151 (1970) and Vol. 22, No. 3, 4, 137149 (1972).

[2] J. J. ERPENBECK : Stability of steady-state equilibrium detonations, The physics of Fluids, Vol. 5, No. 5, 604-614 (1962).

[3] J. J. ERPENBECK: Stability of step shocks, The Physics of Fluids, Vol. 5, No. 10, 1181-1187 (1962).

[4] L. HöRMANDER: Pseudo-differential operators and non-elliptic boundary problems, Ann. Math., Vol. 83, 130-209 (1966).

[5] L. D. LAndau-E. M. Lifshitz: Fluid Mechanics, Course of Theoretical Physics, Vol. 6, Pergamon Press. 1966.

[6] P. D. LAX : Hyperbolic systems of conservation laws, II, Comm. pure Appl. Math., Vol. 10, 537-556 (1957).

[7] A. MAJDA: The stability of multi-dimensional shock fronts -a new problem for linear hyperbolic eguations, (to appear).

[8] A. MAJDA: The existence of multi-dimensional shock fronts, (to appear).

[9] V. P. MASLOV: Propagation of shock waves in an isentropic nonviscous gas, Sovrem. Probl. Math., Vol. 8, 199-271 (1977). (J. Soviet Mate., Vol. 13, No. 1, 119-162 (1980).)

[10] T. OKubo-T. Shirota: On structures of $L^{2}$-well posed mixed problems for hyperbolic systems of first order, Hokkaido Math. J., Vol. 4, 82-158 (1975).

[11] J. RAUCH : Energy inequalities for hyperbolic initial boundary value problems, Thesis, New York Univ., February, (1981).

[12] T. SHIROTA: On the propagation speed of hyperbolic operator with mixed boundary conditions, J. Fac. Sci. Hokkaido Univ., Ser. I, Vol. 22, 25-31 (1972).

[13] K. S. ECKHOFF-L. STOREsletten: A note on the stability of steady inviscide helical gas flows, J. Fluid Mech., Vol. 89, part 3, 401-411 (1978).

[14] K. S. EchкHOFF: On stability of symmetric hyperbsolic sytems, 1, J. Diff. Eq., Vol. 40, 94-115 (1981). 